\title{
Chemical Mapping of Ceramide Distribution in Sphingomyelin-Rich Domains in Monolayers
}

\author{
Jesse Popov, ${ }^{\dagger}$ Dusan Vobornik,${ }^{\dagger}$ Oana Coban, ${ }^{\dagger}$ Eleonora Keating, ${ }^{\S}$ David Miller, ${ }^{\S}$ \\ James Francis, ${ }^{\S}$ Nils O. Petersen, ${ }^{\ddagger}$ and Linda J. Johnston ${ }^{* \dagger}$ \\ Steacie Institute for Molecular Sciences, National Research Council of Canada, 100 Sussex Drive, Ottawa, \\ Ontario, Canada K1A OR6, National Institute for Nanotechnology, National Research Council of Canada, \\ Edmonton, Alberta, Canada, and Department of Chemistry, University of Western Ontario, London, \\ Ontario, Canada
}

Received March 12, 2008. Revised Manuscript Received August 29, 2008

\begin{abstract}
The incorporation of ceramide in phase-separated monolayers of ternary lipid mixtures has been studied by a combination of atomic force microscopy (AFM), fluorescence, and time-of-flight secondary ion mass spectrometry (ToF-SIMS). Replacement of a fraction of the sphingomyelin by ceramide in DOPC/SM/cholesterol monolayers leads to changes in the SM-cholesterol-rich liquid-ordered domains. AFM shows the formation of heterogeneous domains with small raised islands that are assigned to a ceramide-rich gel phase. ToF-SIMS provides conclusive evidence for the localization of SM and ceramide in ordered domains and shows that ceramide is heterogeneously distributed in small islands throughout the domains. The results indicate the utility of combining AFM and ToF-SIMS for understanding compositions of phase-separated membranes.
\end{abstract}

\section{Introduction}

Ceramides are among the most nonpolar and hydrophobic lipids found in cell membranes. They are involved in various signaling pathways and are thought to be second messengers in cellular processes such as apoptosis, senescence, bacterial pathogenesis, and cell cycle arrest. ${ }^{1-3}$ Some studies have indicated that ceramide acts as a second messenger by binding to specific intracellular protein targets, whereas others have concluded that its function as a signaling molecule is mediated through its strong impact on the biophysical properties of membranes. These effects include the ability of ceramide to increase lipid order, form ceramide-rich domains, promote the formation of nonlamellar structures, ${ }^{4,5}$ and induce transbilayer flip-flop. ${ }^{4,6,7}$

Ceramides are formed by either de novo synthesis or enzymecatalyzed hydrolysis of sphingomyelin (SM), one of the main components of membrane rafts. ${ }^{8}$ Rafts are small $(10-200 \mathrm{~nm})$, heterogeneous, dynamic, cholesterol (chol), and sphingolipidenriched domains that compartmentalize cellular processes. ${ }^{9}$ They are generally believed to exist in a liquid-ordered phase with properties distinct from the surrounding fluid disordered membrane. ${ }^{10-14}$ Both lipids and proteins contribute to the formation of these membrane domains and their functional role in

\footnotetext{
${ }^{\dagger}$ National Research Council of Canada, Ottawa.

* National Research Council of Canada, Edmonton.

${ }^{\S}$ University of Western Ontario.

(1) Cremesti, A. E.; Goni, F. M.; Kolesnick, R. FEBS Lett. 2002, 531, 47-53.

(2) Bollinger, C. R.; Teichgraber, V.; Gulbins, E. Biochim. Biophys. Acta 2005, 1746, 284-294.

(3) Goni, F. M.; Alonso, A. Biochim. Biophys. Acta 2006, 1758, 1902-1921.

(4) Contreras, F. X.; Villar, A. V.; Alonso, A.; Kolesnick, R. N.; Goni, F. M. J. Biol. Chem. 2003, 278, 37169-74.

(5) Kolesnick, R. N.; Goni, F. M.; Alonso, A. J Cell Physiol. 2000, 184, 285-300.

(6) Lopez-Montero, I.; Rodriguez, N.; Cribier, S.; Pohl, A.; Velez, M.; Devaux, P. F. J. Biol. Chem. 2005, 280, 25811-25819.

(7) Contreras, F. X.; Basanez, G.; Alonso, A.; Herrmann, A.; Goni, F. M. Biophys. J. 2005, 88, 348-59.

(8) Goni, F. M.; Alonso, A. FEBS Lett. 2002, 531, 38-46.

(9) Pike, L. J. J. Lipid Res. 2006, 47, 1597-1598.

(10) Brown, D. A.; London, E. Annu. Rev. Cell Dev. Biol. 1998, 14, 111-136.

(11) Edidin, M. Annu. Rev. Biophys. Biomol. Struct. 2003, 32, 257-283.

(12) Laude, A.; Prior, I. A. Mol. Memb. Biol. 2004, 21, 193-205.

(13) Simons, K.; Ikonen, E. Nature 1997, 387, 569-572.
}

modulating cellular processes via both the spatial and temporal compartmentalization of membrane components is an important part of the raft definition. Conversion of raft sphingomyelin to ceramide by sphingomyelinase (SMase) has been suggested to cause coalescence of small rafts to give larger signaling platforms, providing sites to oligomerize cell-surface receptors, to internalize bacteria, and to aid in the inhibition of HIV invasion of cells. ${ }^{2,15}$ It has also been hypothesized that ceramide displaces chol from rafts in model ${ }^{16-19}$ and cellular membranes. ${ }^{20}$

The influence of ceramide on supported bilayers and vesicles has been examined using a range of microscopic techniques. For example, treating phosphatidylcholine/SM giant vesicles with SMase led to formation of ceramide patches that coalesced into ceramide-rich macrodomains and then formed vesicles. ${ }^{21,22}$ Ceramide-enriched domains have also been observed in phospholipid vesicles, ${ }^{23,24}$ in supported bilayers ${ }^{25}$ and in SM/ceramide giant unilamellar vesicles. ${ }^{26}$ Other work has shown that direct incorporation of ceramide in supported bilayers with ordered SM-chol-rich domains results in formation of micrometer-sized ordered domains containing small higher islands that are assigned

(14) Simons, K.; Vaz, W. L. Annu. Rev. Biophys. Biomol. Struct. 2004, 33, 269-295.

(15) Grassme, H.; Jendrossek, V.; Riehle, A.; von Kurthy, G.; Berger, J.; Schwarz, H. Weller, M. Kolesnick, R.; Gulbins, E. Nat Med. 2003, 9, 322-30. (16) Megha, London, E. J. Biol. Chem. 2004, 279, 9997-10004.

(17) Megha, Bakht, O.; London, E. J. Biol. Chem. 2006, 281, 21903-21913. (18) Chiantia, S.; Kahya, N.; Ries, H.; Schwille, P. Biophys. J. 2006, 90, $4500-4508$.

(19) Ali, M. R.; Cheng, K. H.; Huang, J. Biochem. 2006, 45, 12629-12638. (20) Yu, C.; Alterman, M.; Dobrowsky, R. T. J. Lipid Res. 2005, 46, 16781691.

(21) Nurminen, T. A.; Holopainen, J. M.; Zhao, H.; Kinnunen, P. K. J. Am. Chem. Soc. 2002, 124, 12129-34.

(22) Holopainen, J. M.; Angelova, M. I.; Kinnunen, P. K. J. Biophys. J. 2000, $78,830-838$.

(23) Silva, L. C.; de Almeida, R. F. M.; Castro, B. M.; Fedorov, A.; Prieto, M. Biophys. J. 2007, 92, 502-516.

(24) Taniguchi, Y.; Ohba, T.; Miyata, H.; Ohki, K. Biochim. Biophys. Acta 2006, 1758, 145-153.

(25) Blanchette, C. D.; Lin, W.-C.; Orme, C. A.; Ratto, T. V.; Longo, M. L. Biophys. J. 2008, 94, 2691-2697.

(26) Sot, J.; Bagatolli, L. A.; Goni, F. M.; Alonso, A. Biophys. J. 2006, 90 , 903-914. 
to a new ceramide-rich phase. ${ }^{18,27}$ Enzymatic generation of ceramide causes pronounced restructuring of supported bilayers, leading to significant changes in size and morphology of initial liquid-ordered raft domains. ${ }^{27,28}$

Ceramide effects on lipid monolayers have also been examined. Langmuir-Blodgett monolayers comprised of ceramides, chol, and fatty acids that are used to model the skin strateum corneum composition exhibit ceramide-rich domains for a variety of lipid ratios. ${ }^{29-31}$ Other studies have examined the influence of ceramide structure and surface pressure on the miscibility of mixed ceramide phosphatidylcholine monolayers. ${ }^{32,33}$ The formation of a ceramide-enriched phase has been observed for chol/ceramide monolayers ${ }^{34}$ and for SM monolayers at the air-water interface after treatment with SMase enzyme. ${ }^{35,36}$ The observation that ceramide promotes phase separation to give ceramide-rich ordered phases is common to most of the bilayer and monolayer studies cited above, although neither lipid miscibility nor domain morphology can be compared directly between the two systems.

Although the addition of ceramide to ternary lipid mixtures that have coexisting fluid and liquid-ordered phases has been investigated in bilayers, it has not yet been examined in monolayers. Our previous studies of monolayers with coexisting ordered SM-chol rich domains surrounded by a fluid DOPC matrix have relied on combinations of atomic force microscopy (AFM) and fluorescence to examine membrane morphology. ${ }^{37,38}$ However, neither technique provides direct information on chemical composition. Furthermore, although area analysis of simple binary lipid mixtures yields lipid composition of monolayer domains, ${ }^{39}$ this is more difficult for three or four component monolayers. Recent developments in chemical mapping using secondary ion mass spectrometry have shown considerable promise for probing the location and amounts of lipid components in domains in supported monolayers and bilayers. ${ }^{40-43}$ Notably, several recent studies have mapped the lipid distribution in mixtures with coexisting fluid and gel or liquid-ordered phases. ${ }^{40,43-45}$ Although mass spectrometry imaging does not yet have the accuracy for determining lipid composition that has

(27) Ira; Johnston, L. J. Langmuir 2006, 22, 11284-11289.

(28) Ira; Johnston, L. J. Biochim. Biophys. Acta 2008, 1778, 185-197.

(29) ten Grotenhuis, E.; Demel, R. A.; Ponec, M.; Boer, D. R.; van Miltenburg, J. C.; Bouwstra, J. A. Biophys. J. 1996, 71, 1389-1399.

(30) Sparr, E.; Eriksson, L.; Bouwstra, J. A.; Ekelund, K. Langmuir 2001, 17, 164-172.

(31) Norlen, L.; Gil, I. P.; Simonsen, A.; Descouts, P. J. Struct. Biol. 2007, $158,386-400$.

(32) Holopainen, J. M.; Brockman, H. L.; Brown, R. E.; Kinnunen, P. K. J. Biophys. J. 2001, 80, 765-775.

(33) Imura, T.; Sakai, H.; Yamauchi, H.; Kozawa, K.; Yokoyama, S.; Matsumoto, M.; Abe, M. Colloids Surf., B 2000, 19, 81-97.

(34) Scheffer, L.; Solomonov, I.; Weygard, M. J.; Kjaer, K.; Leiserowitz, L.; Addadi, L. Biophys. J. 2005, 88, 3381-3391.

(35) Fanani, M. L.; Hartel, S.; Oliveira, R. G.; Maggio, B. Biophys. J. 2002, $83,3416-3424$

(36) Hartel, S.; Fanani, M. L.; Maggio, B. Biophys. J. 2005, 88, 287-304. (37) Coban, O.; Popov, J.; Burger, M.; Vobornik, D.; Johnston, L. J. Biophys. J. 2007, 92, 2842-2853.

(38) Coban, O.; Burger, M.; Laliberte, M.; Ianoul, A.; Johnston, L. J. Langmuir 2007, 23, 6704-6711.

(39) Hagen, J. P.; McConnell, H. M. Biochim. Biophys. Acta 1997, 1329, $7-11$.

(40) Kraft, M. L.; Weber, P. K.; Longo, M. L.; Hutcheon, I. D.; Boxer, S. G. Science 2006, 313, 1948-1951.

(41) Winograd, N. Anal. Chem. 2005, 77, 143A-149A.

(42) Keating, E.; Rahman, L.; Francis, J.; Petersen, A.; Possmayer, F.; Veldhuizen, R.; Petersen, N. O. Biophys. J. 2007, 93, 1391-1401.

(43) McQuaw, C. M.; Zheng, L.; Ewing, A. G.; Winograd, N. Langmuir 2007, $23,5645-5650$

(44) Sostarecz, A. G.; McQuaw, C. M.; Ewing, A. G.; Winograd, N. J. Am. Chem. Soc. 2004, 126, 13882-13883.

(45) Zheng, L.; McQuaw, C. M.; Ewing, A. G.; Winograd, N. J. Am. Chem. Soc. 2007, 129, 15730-15731. been reported for NMR techniques, ${ }^{46}$ it provides the advantage of direct visualization of domains as well as information on their lipid composition. Herein, we use AFM, fluorescence microscopy and time-of-flight secondary ion mass spectrometry (ToF-SIMS) imaging to examine the morphologies of DOPC/SM/chol monolayers in which varying fractions of SM have been replaced by ceramide. The combination of methods allows the direct visualization of the effects of ceramide on SM-chol-rich domains and the correlation of monolayer morphologies with lipid composition. Ceramide addition leads to heterogeneous liquidordered domains with small raised subdomains that are shown by ToF-SIMS to be ceramide-enriched regions.

\section{Materials and Methods}

Materials. Dioleoylphosphatidylcholine (DOPC), egg SM

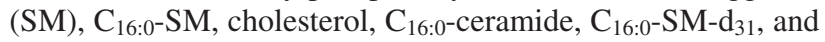
$\mathrm{C}_{16: 0}$-ceramide- $\mathrm{d}_{31}$ were purchased from Avanti Polar Lipids and were used as received. DOPC was purchased as a chloroform solution at a concentration of $10 \mathrm{mg} / \mathrm{ml}$ in ampule sealed under nitrogen and was stored at $-20^{\circ} \mathrm{C}$. All other lipids were stored as lyophilized powders at $-20^{\circ} \mathrm{C}$. HPLC grade chloroform and methanol were from ACP Chemicals. Texas Red 1,2-dihexadecanoyl-sn-glycero-3-phosphoethanolamine, triethylammonium salt (Texas Red DHPE) was purchased from Invitrogen Canada and 1,1'-dioctadecyl-3,3,3',3'-tetramethylindocarbocyanine perchlorate (DiI- $\mathrm{C}_{18: 0}$ ) from Molecular Probes.

Monolayer Preparation. Planar supported monolayers were prepared on a Langmuir-Blodgett trough (611 NIMA) using deionized water $(18.3 \mathrm{M} \Omega \cdot \mathrm{cm})$ as the subphase. To prevent oxidation of unsaturated lipids on the surface of the water subphase, the LB trough was placed in a glovebox (Plas Laboratories 830-Compact Glove Box) filled with nitrogen, which ensured an inert environment for monolayer preparation. Fresh stock solutions of lipid were prepared in chloroform before each sample preparation. A new ampule of DOPC was opened for each experiment. Lipid mixtures of the desired composition (ratios in $\mathrm{mol} \%$ ) were mixed and spread on the water surface at $\sim 5$ $\mathrm{mN} / \mathrm{m}$ and allowed to equilibrate to a constant surface pressure upon solvent evaporation $(2-5 \mathrm{~min})$. The surface pressure was measured with a precision of $0.1 \mathrm{mN} / \mathrm{m}$ using a Wilhelmy balance. Monolayers were annealed by at least two compression/expansion cycles at $150 \mathrm{~cm}^{2} / \mathrm{min}$ and once again at $75 \mathrm{~cm}^{2} / \mathrm{min}$. Monolayers were transferred to freshly cleaved mica (AFM and NSOM) or gold-coated mica (ToF-SIMS) at a preset pressure by vertical deposition at a dipping speed of $2 \mathrm{~mm} / \mathrm{min}$. Monolayers were stored under nitrogen until imaged. In previous work, no change in monolayer morphology, as assessed by AFM images, has been observed for monolayers stored in either nitrogen or air for several days. ${ }^{37}$

Scanning Probe Microscopy. AFM measurements were performed on a Digital Instruments Multimode Nanoscope III in contact mode in air. Monolayers were scanned at constant force using a piezoelectric $\mathrm{J}$ scanner (maximum scan area 120 $\left.\times 120 \mu \mathrm{m}^{2}\right)$ and soft cantilevers ( $200 \mu \mathrm{m}$ in length) with integrated pyramidal tips (spring constant $\sim 120 \mathrm{mN} / \mathrm{m}$ ). Usually, regions ranging from $100 \times 100 \mu \mathrm{m}^{2}$ to $5 \times 5 \mu \mathrm{m}^{2}$ were imaged at a resolution of $512 \times 512$ pixels using a scan rate of $1 \mathrm{~Hz}$. Multiple samples (a minimum of three) were prepared and imaged for all monolayer compositions. Differences in height between coexisting phases of the monolayers were determined using the cross-section analysis routine of the DI software. The heights listed in the text are obtained by analyzing a minimum of three

(46) Veatch, S. L.; Gawrisch, K.; Keller, S. L. Biophys. J. 2006, 90, 4428- 
images for the samples shown in the figures. The surface area covered by the two phases within the domains was analyzed using ImagePro software and the data refer to the specific images shown in the figures.

NSOM experiments were carried out on a combined AFM/ NSOM microscope. The NSOM setup is based on a Digital Instruments Bioscope mounted on an inverted microscope (Axiovert $100 \mathrm{TV}, \mathrm{Zeiss}$ ) using an $\mathrm{Ar}^{+}-\mathrm{Kr}^{+}$ion laser (Coherent, Innova 70 Spectrum) as the excitation source. The bent NSOM probe is fabricated from single mode optical fiber using a twostep chemical etching method followed by aluminum deposition and focused ion beam milling to produce the aperture. ${ }^{47}$ All experiments were performed with a tip aperture size of $<100 \mathrm{~nm}$ and estimated spring constant of $100 \mathrm{~N} / \mathrm{m}$. A more detailed description of the probe fabrication and characterization is given elsewhere. ${ }^{47,48}$ Fluorescence is collected through an air objective (Zeiss Ldplan-Neofluar, $63 \times, \mathrm{NA}=0.75$ ) passed through an appropriate notch filter to remove residual excitation light and a band-pass filter and detected by an avalanche photodiode (EG\&G, SPCM-AQR-14). Images were collected in tapping mode in air at a scan rate of typically $0.25 \mathrm{~Hz}$ and a resolution of 512 $\times 512$ pixels.

ToF-SIMS. An ION-TOF (Gmbh) ToF-SIMS IV equipped with a bismuth liquid metal ion source was employed in this study. The primary analysis beam was $25 \mathrm{keV} \mathrm{Bi}_{3}{ }^{+}$operated in the Burst Alignment mode ${ }^{49}$ with a $10 \mathrm{kHz}$ repetition rate and a pulse width of $100 \mathrm{~ns}$. The target current was $<0.2 \mathrm{pA}$ and the spot size was estimated to be $\sim 300 \mathrm{~nm}$. Mass analysis was performed via a single stage reflectron ToF analyzer, at $2 \mathrm{keV}$ with $10 \mathrm{keV}$ post acceleration. The mass range was $0-800 \mathrm{amu}$, with unit mass resolution. Negative secondary ion images were acquired from regions of interest, typically with a $50 \times 50 \mu \mathrm{m}^{2}$ primary beam raster size. A $128 \times 128$ pixel image resolution was chosen, given the beam spot size and the raster area used in this work. The negative secondary ion images of interest were reconstructed from the raw data stream. Each image is normalized in intensity according to the minimum and maximum counts in a single pixel; these values are then mapped to a 256 increment, thermal color scale. The thermal gradient is chosen such that brighter areas in an image correspond to areas of increased secondary ion yield. In some cases the ToF-SIMS images were processed to enhance contrast by using a smoothing function available as part of the ION-TOF software. Either two or three separate samples were imaged for each sample composition, with multiple areas imaged for each sample.

\section{Results}

AFM and Fluorescence Imaging. Ternary lipid mixtures composed of DOPC/SM/chol phase separate to give coexisting fluid and liquid-ordered phases over a range of compositions in both monolayer and bilayer membranes. ${ }^{50,51}$ Here, we prepared $\mathrm{DOPC} / \mathrm{SM} / \mathrm{chol}$ (40:40:20 molar ratio) monolayers in the presence of variable concentrations of ceramide. In all cases, a fraction of the ESM was replaced with ceramide so that the mol fraction of ESM and ceramide was maintained constant at $40 \%$. Isotherms recorded in the presence of 0 to $20 \%$ ceramide showed that replacement of SM by ceramide led to a gradual decrease of the average molecular area of the monolayer, with area/molecule

(47) Burgos, P.; Lu, Z.; Ianoul, A.; Hnatovsky, C.; Viriot, M.-L.; Johnston, L. J.; Taylor, R. S. J. Microsc. 2003, 211, 37-47.

(48) Ianoul, A.; Street, M.; Grant, D.; Pezacki, J.; Taylor, R.; Johnston, L. J. Biophys. J. 2004, 87, 3525-3535.

(49) Sodhi, R. N. S. Analyst 2004, 129, 483-487.

(50) Veatch, S. L.; Keller, S. L. Biochim. Biophys. Acta 2005, 1746, 172-185.

(51) Johnston, L. J. Langmuir 2007, 23, 5886-5895.

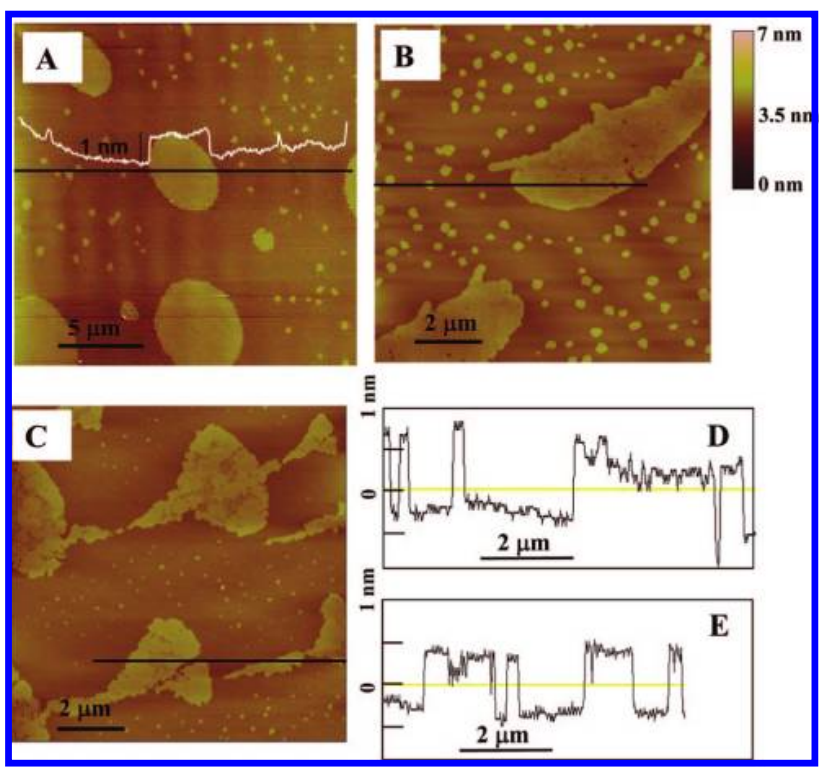

Figure 1. AFM images showing the effect of replacing SM by ceramide in $\mathrm{DOPC} / \mathrm{SM} / \mathrm{chol} /$ ceramide monolayers deposited at $10 \mathrm{mN} / \mathrm{m}$. The DOPC/SM/chol/ceramide ratios are 40:40:20:0 (A), 40:30:20:10 (B), and 40:20:20:20(C). The cross sections show heights of the heterogeneous domains observed in the presence of 10 (D) and 20\% (E) ceramide.

values ranging from 49 to $40 \AA^{2}$ for 0 and $20 \%$ ceramide respectively at a surface pressure of $30 \mathrm{mN} / \mathrm{m}$ (Figure $\mathrm{S} 1$ of the Supporting Information). A similar effect has been observed upon addition of ceramide to phosphatidylcholine and SM monolayers. . $^{32,33,35}$

Monolayers containing variable ceramide concentrations were transferred to mica at two different surface pressures, 10 and 30 $\mathrm{mN} / \mathrm{m}$. These two pressures were chosen for comparison with our previous study of DOPC/SM/chol monolayers: $;{ }^{37}$ furthermore, a pressure of $30 \mathrm{mN} / \mathrm{m}$ is within the estimated range for modeling a biological membrane. ${ }^{52,53}$ Parts $\mathrm{A}-\mathrm{C}$ of Figure 1 show the effect of ceramide addition for monolayers transferred to mica at a surface pressure of $10 \mathrm{mN} / \mathrm{m}$. In the absence of ceramide (part A of Figure 1), the monolayer has a mixture of large and small domains that are $0.90 \pm 0.06 \mathrm{~nm}$ higher than the surrounding fluid phase, as reported previously. ${ }^{37}$ By analogy to earlier studies, the domains are assigned to a SM-chol-rich phase, which is surrounded by a DOPC-rich liquid-disordered phase. ${ }^{54-56}$ In the presence of $10 \%$ ceramide (DOPC/SM/chol/cer ratio of 40:30: 20:10, Part B of Figure 1), the monolayer morphology is qualitatively similar with a mixture of small and large raised domains; however, the large ordered domains are heterogeneous in height as shown in the cross section in part D of Figure 1. The edges of the domains are $0.94 \pm 0.07 \mathrm{~nm}$ higher than the surrounding lower phase, whereas the interior of the domains has some regions that are the same height as the edges and other lower areas, $0.5 \pm 0.2 \mathrm{~nm}$. The broad distribution of domain heights throughout the domains is in contrast to monolayers in the absence of ceramide as shown by to the cross section in part A of Figure 1. Similarly, monolayers containing $20 \%$ ceramide (part C of Figure 1) also show heterogeneous domains with two distinct heights, $0.92 \pm 0.08$ and $0.5 \pm 0.1 \mathrm{~nm}$ above the lower

(52) Feng, S.-S. Langmuir 1999, 15, 998-1010

(53) Nagle, J. F. J. Membr. Biol. 1976, 27, 233-250.

(54) Milhiet, P. E.; Domec, C.; Giocondi, M.-C.; Mau, N. V.; Heitz, F.; Le Grimellec, C. Biophys. J. 2001, 81, 547-555.

(55) Yuan, C.; Johnston, L. J. J. Microsc. 2002, 205, 136-146.

(56) Lawrence, J. C.; Saslowsky, D. E.; Edwardson, J. M.; Henderson, R. M. Biophys. J. 2003, 84, 1827-1832. 


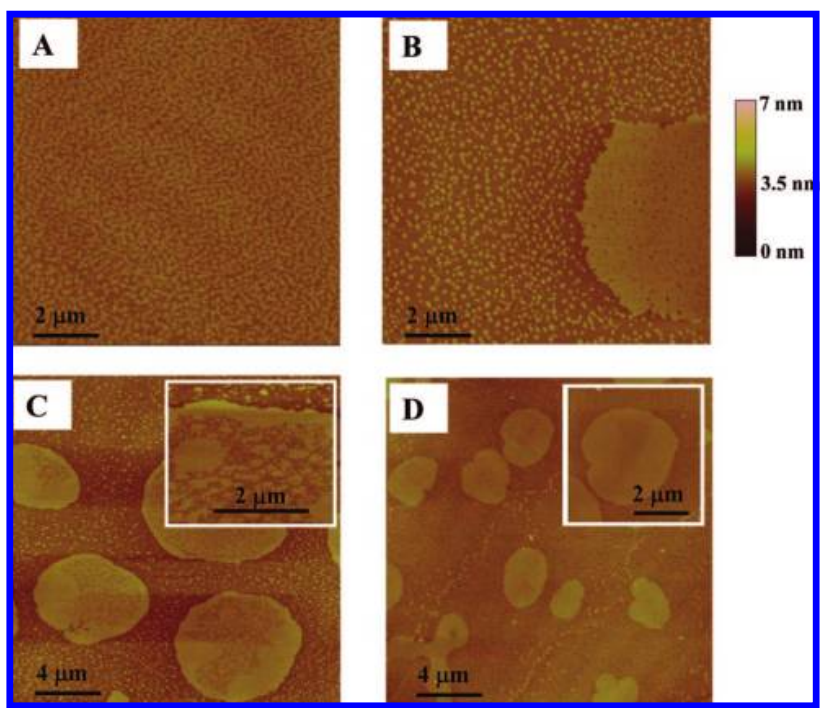

Figure 2. AFM images showing the effect of replacing SM by ceramide in DOPC/SM/chol/ceramide monolayers deposited at $30 \mathrm{mN} / \mathrm{m}$. The DOPC/SM/chol/ceramide ratios are 40:40:20:0 (A), 40:38:20:2 (B), 40: 30:20:10 (C), and 40:20:20:20 (D). The small images in the inserts in $\mathrm{C}$ and $\mathrm{D}$ clearly illustrate the heterogeneity of individual domains. The $\mathrm{Z}$-scale is $7 \mathrm{~nm}$ for all images except $\mathrm{C}$, which is displayed on a $\mathrm{Z}$-scale of $5 \mathrm{~nm}$.

phase (part E of Figure 1). The higher regions of the domains for the monolayer containing $20 \%$ ceramide account for $80 \%$ of the total domain surface area, as compared to $57 \%$ for the $10 \%$ ceramide monolayer shown in part B of Figure 1. Note that in the presence of ceramide the domain boundaries are irregular, indicative of a more gel-like ordered phase. Monolayers containing $2 \%$ ceramide also showed domains with multiple heights (data not shown).

Figure 2 shows a similar set of experiments for monolayers deposited at higher surface pressure $(30 \mathrm{mN} / \mathrm{m})$. In this case, the monolayer in the absence of ceramide shows small domains, some with irregular, elongated, and branched shapes. Previous studies have shown that this DOPC/SM/chol mixture undergoes a transition from a mixture of large and small domains at low surface pressure to small domains above $\sim 25 \mathrm{mN} / \mathrm{m} .{ }^{37}$ The addition of even $2 \%$ ceramide is sufficient to change the monolayer morphology dramatically (part B of Figure 2), giving a mixture of large and small domains that are similar to those obtained at low surface pressure. With $2 \%$ ceramide the large ordered domains are uniform in height $(0.8 \pm 0.1 \mathrm{~nm}$ above the lower phase $)$, except for the presence of small islands $\left(\sim 20\right.$ islands $\left./ \mu \mathrm{m}^{2}\right)$ of the lower, fluid phase. By contrast, images for monolayers containing 10\% ceramide (part C of Figure 2) show large ordered domains that have two distinct domain heights, $0.72+0.09$ and $0.4 \pm 0.1 \mathrm{~nm}$. The higher areas are found both on the domain edges and interior, whereas the lower height areas are within the domain interior. With $20 \%$ ceramide (part D of Figure 2) there are both irregular-shaped domains (lower left corner of image) and others that are close to circular or elliptical shapes. The domains have two heights, $0.72+0.09$ and $0.4 \pm 0.1 \mathrm{~nm}$, with the higher phase predominantly around the edges and the lower phase in the interior. The higher parts of the domain account for 64 and $80 \%$ of the total domain surface area for 10 and $20 \%$ ceramide, respectively, on the basis of the images shown in parts $\mathrm{C}$ and $\mathrm{D}$ of Figure 2.

Several monolayers were examined using near field scanning optical microscopy (NSOM) in an attempt to provide more details on the complex domain morphology observed for ceramide

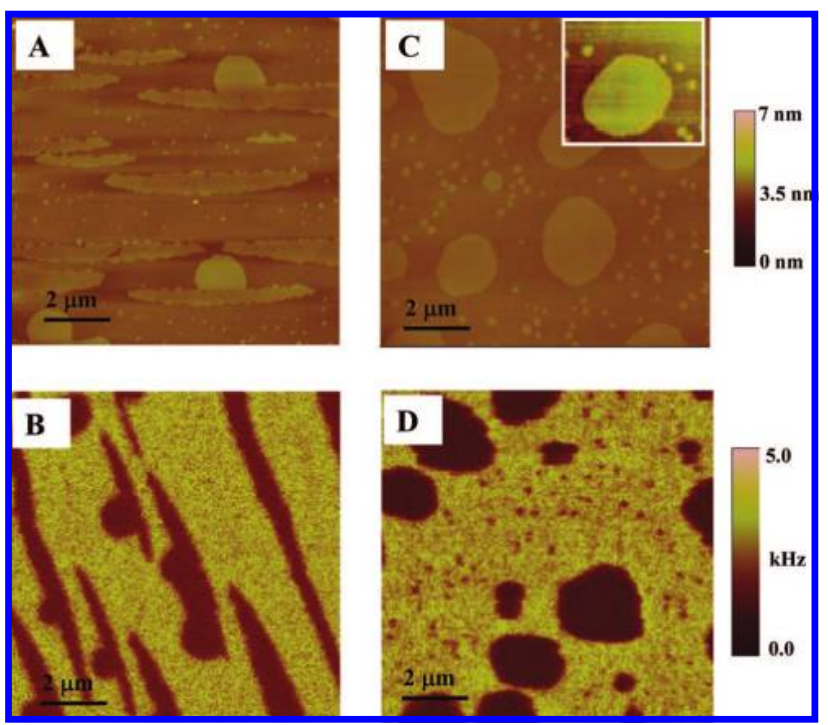

Figure 3. AFM (A, C) and NSOM (B, D) images for 40:20:20:20 DOPC/ $\mathrm{SM} / \mathrm{chol} /$ ceramide monolayers containing 1\% Texas Red-DHPE and deposited at $10 \mathrm{mN}(\mathrm{A}, \mathrm{B})$ and $30 \mathrm{mN}(\mathrm{C}, \mathrm{D})$. The z-scale for the inset in image $C$ is $4 \mathrm{~nm}$ in order to observe the domain heterogeneity; large AFM images have a $\mathrm{z}$-scale of $7 \mathrm{~nm}$.

containing monolayers. Figure 3 shows a comparison of AFM and near field fluorescence images for two monolayers prepared with 20\% ceramide and using 1\% Texas Red-DHPE for fluorescence visualization. The addition of dye slightly modifies the monolayer morphology at $10 \mathrm{mN} / \mathrm{m}$ (part A and B of Figure 3 ); there are still two distinct domain heights but the higher of the two now occurs as round areas adjacent to lower height elliptical domains. NSOM images show that dye is excluded from both types of domains. Similarly, dye is excluded from the heterogeneous domains obtained for monolayers containing $20 \%$ ceramide deposited at $30 \mathrm{mN} / \mathrm{m}$ (parts C and D of Figure 3). Note that the high spatial resolution of the near field microscope allows one to clearly visualize the small closely spaced islands that would not be detectable by diffraction-limited techniques and indicates that these are an ordered phase, similar to the large domains. A similar experiment was carried out with DiI C $18: 0$, which has been reported to partition into liquid-ordered phases in some ternary lipid mixtures ${ }^{57}$ However, this dye also showed strong partitioning into the lower, fluid phase. Although the fluorescence results do not provide any information on the differences in fluidity of the raised regions within the domains in the presence of ceramide, they are consistent with the domains being in a more ordered gel-like phase after ceramide incorporation.

ToF-SIMS Imaging of Lipid Composition. Monolayers containing ceramide were imaged by ToF-SIMS to provide information on the chemical composition of the heterogeneous domains observed by AFM. Although reference spectra for films of the individual lipids in both positive and negative ion mode gave characteristic molecular ion peaks, a DOPC/SM/chol (40: 40:20) monolayer did not show strong molecular ions (Figure S2 in Supporting Information). On the basis of this and the difficulty of finding a characteristic fragment that could be used to distinguish between SM, ceramide and DOPC, we employed ceramide and SM with a single fully deuterated acyl chain $\left(\mathrm{d}_{31}\right)$ to provide a characteristic signal that could be traced to a specific lipid. This gives a mass peak at $m / z 2$ due to deuterium in negative

(57) Baumgart, T.; Hunt, G.; Farkas, E. R.; Webb, W. W.; Feigenson, G. W. Biochim. Biophys. Acta 2007, 1768, 2182-2194. 


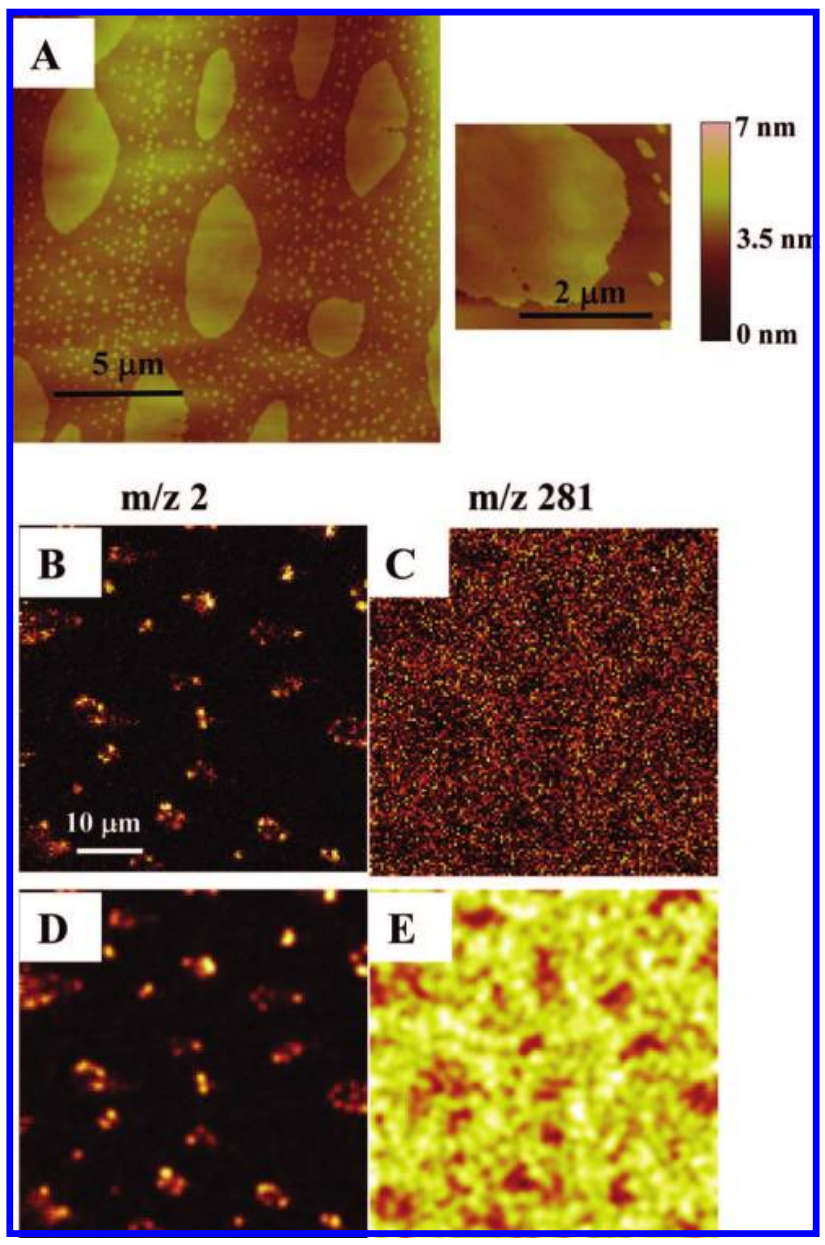

Figure 4. AFM (A) and ToF-SIMS (B-E) images for 40:30:20:10 DOPC/ $\mathrm{SM} / \mathrm{chol} /$ ceramide- $d_{31}$ monolayers deposited at $10 \mathrm{mN} / \mathrm{m}$. Images $\mathrm{D}$ and $\mathrm{E}$ are obtained after smoothing of the $m / z 2$ and 281 images shown in B and C, respectively. The pixel size for images B-E is $404 \mathrm{~nm}$, and the experimental resolution is estimated to be $\sim 300 \mathrm{~nm}$.

ion mode that is used to monitor the location of deuterated SM or ceramide. The $\mathrm{m} / \mathrm{z} 281$ signal due to the oleate fragment $\left(\mathrm{CH}_{3}\left(\mathrm{CH}_{2}\right)_{7} \mathrm{CHCH}-\right.$

$\left(\mathrm{CH}_{2}\right)_{7} \mathrm{CO}_{2}^{-}$) was used to monitor DOPC on the basis of earlier results showing that fatty acid fragments provide characteristic signals for identification of phosphatidylcholines. ${ }^{42,58}$ For chol, distinct $m / z 369\left(\mathrm{M}-\mathrm{OH}^{+}\right)$and $386\left(\mathrm{M}-\mathrm{H}^{+}\right)$peaks were observed in positive detection mode for pure films but were not detectable for DOPC/SM/chol (40:40:20 molar ratio) monolayers. Similarly, we were unable to detect a deuterium signal at $\mathrm{m} / z 2$ for chol- $d_{7}$ in negative ion mode, although this approach has been successful for monolayers containing $30 \%$ chol. $^{42}$

Prior to ToF-SIMS imaging, we showed that similar domain morphologies were obtained when $\mathrm{C}_{16: 0}$-ceramide- $d_{31}$ was substituted for ceramide in samples containing $10 \%$ ceramide. Figures 4 and 5 show a comparison of AFM and ToF-SIMS negative ion images for samples containing $10 \%$ ceramide- $d_{31}$ and deposited at 10 and $30 \mathrm{mN} / \mathrm{m}$, respectively. The AFM images (part A of Figure 4 and part A of 5) show heterogeneous domains that are similar to those obtained for unlabeled ceramide. Note that the monolayers are deposited on mica and gold coated mica for the AFM and ToF-SIMS experiments respectively, although we do not see any change in overall monolayer morphology.

(58) Harbottle, R. R.; Nag, K.; McIntyre, N. S.; Possmayer, F.; Petersen, N. O. Langmuir 2003, 19, 3698-3704.

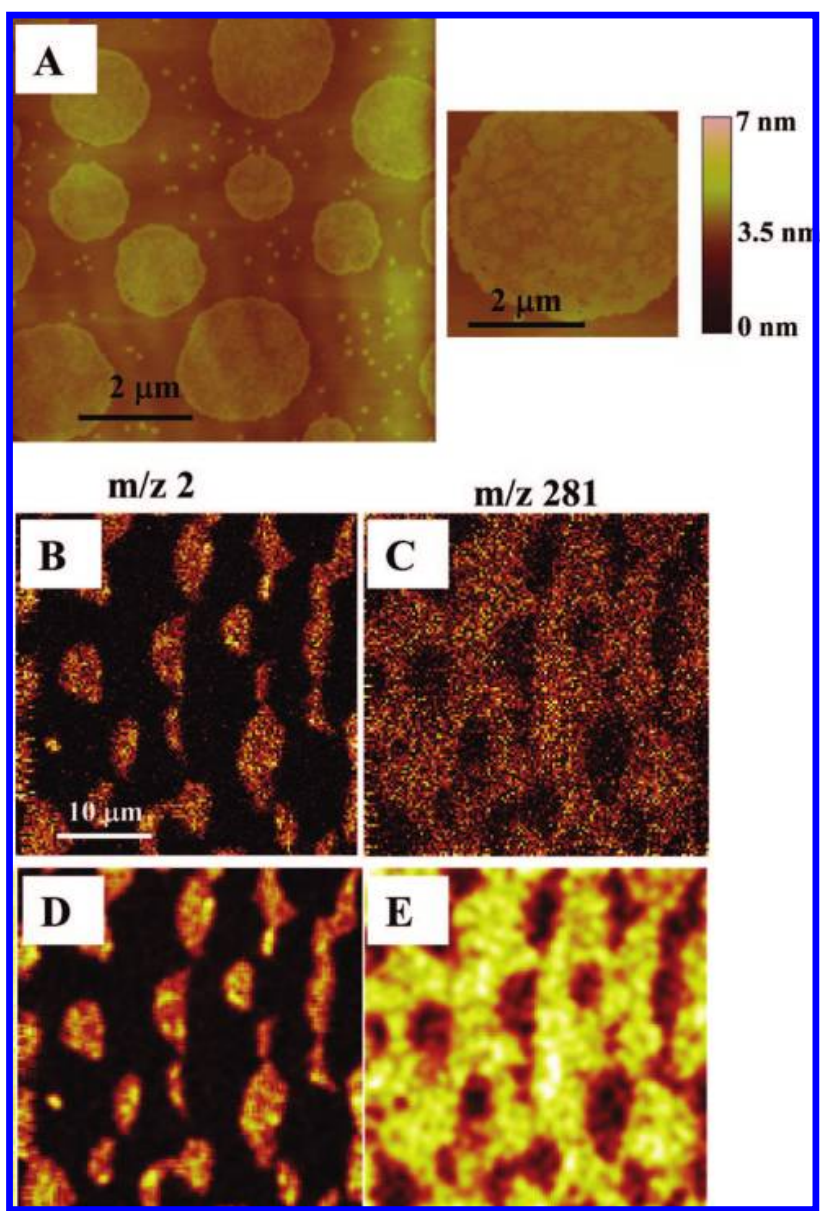

Figure 5. AFM (A) and ToF-SIMS (B-E) images for 40:30:20:10 DOPC/ $\mathrm{SM} / \mathrm{chol} /$ ceramide- $d_{31}$ monolayers deposited at $30 \mathrm{mN} / \mathrm{m}$. Images $\mathrm{D}$ and $E$ are obtained after smoothing of the $m / z 2$ and 281 images shown in $\mathrm{B}$ and $\mathrm{C}$, respectively. The pixel size for images $\mathrm{B}-\mathrm{E}$ is $275 \mathrm{~nm}$, and the experimental resolution is estimated to be $\sim 300 \mathrm{~nm}$.

ToF-SIMS images for a monolayer containing $10 \%$ ceramide- $d_{7}$ and deposited at $10 \mathrm{mN} / \mathrm{m}$ are shown in parts B and C of Figure 4. The deuterium signal at $\mathrm{m} / \mathrm{z} 2$ indicates that ceramide is not distributed uniformly throughout the sample but is concentrated in small islands within elliptical-shaped areas that resemble the raised domains observed by AFM. A ToF-SIMS image for the oleate fragment due to DOPC at $m / z 281$ also shows the presence of domains (dark areas in part C of Figure 4), although the contrast between domains and the surrounding DOPC-rich phase is low compared to the $m / z 2$ signal. Processed images which have been smoothed (parts D and E of Figure 4; see Materials and Methods for details) provide clearer definition of the domains for the oleate signal; the deuterium signal due to ceramide is similar before and after image processing. Comparison of the images for $\mathrm{m} / \mathrm{z} 2$ and 281 provides a clear visualization of ceramide-enriched regions within the individual domains.

Analogous results were obtained for samples containing 10\% ceramide- $d_{31}$ and deposited at $30 \mathrm{mN} / \mathrm{m}$ (Figure 5). Comparison of $\mathrm{m} / \mathrm{z}, 2$ and $\mathrm{m} / \mathrm{z} 281$ signals (parts B and C of Figure 5) again shows that ceramide is unevenly distributed in small subdomains throughout the large ordered domains. In this case, the dark domains for the oleate signal at $\mathrm{m} / \mathrm{z}, 281$ are clearly visible before signal processing (parts D and E of Figure 5). A similar experiment for a monolayer with $10 \%$ ceramide (undeuterated) and $30 \%$ $\mathrm{C}_{16: 0}-\mathrm{SM}-d_{31}$ deposited at $30 \mathrm{mN} / \mathrm{m}$ is shown in Figure 6. The image in part A of Figure 6 indicates that the deuterium signal 


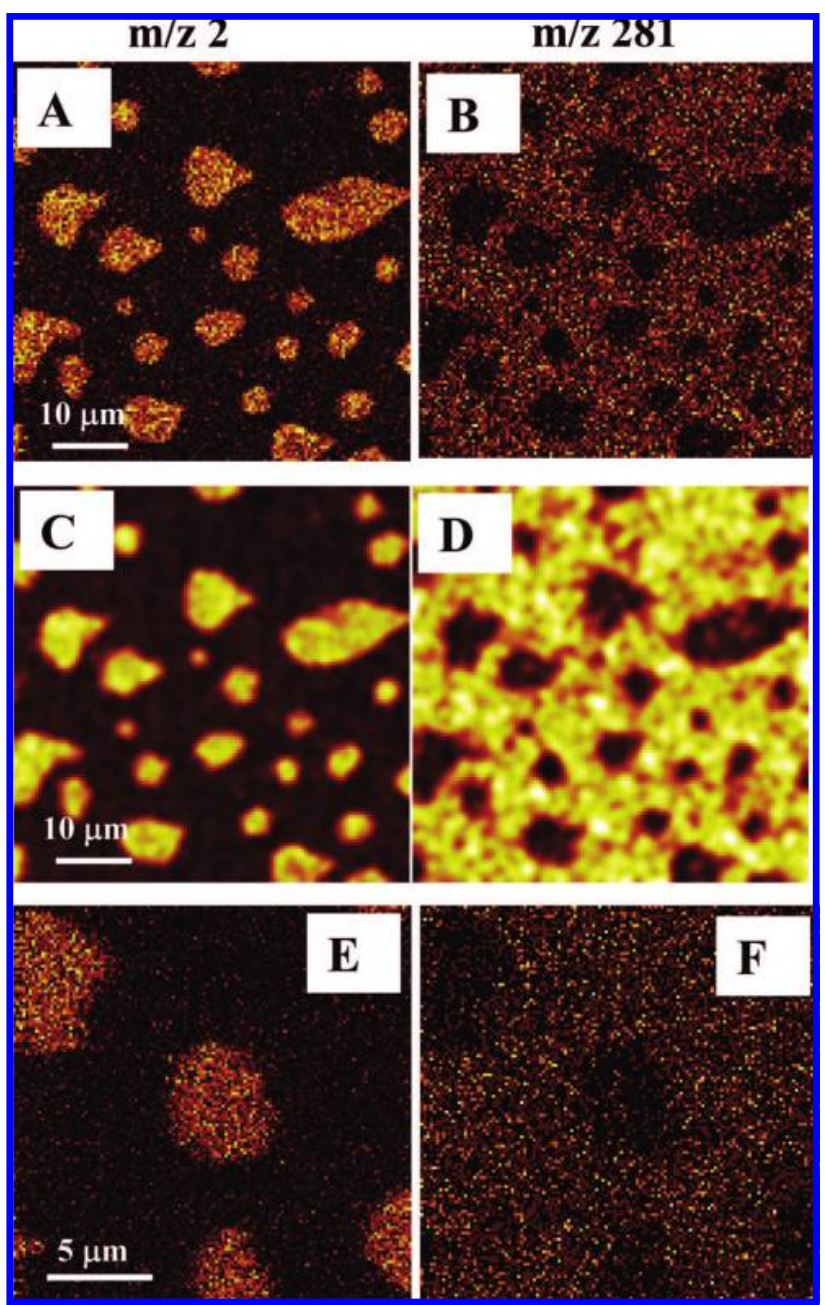

Figure 6. ToF-SIMS images for 40:30:20:10 DOPC/SM- $d_{31} / \mathrm{chol} /$ ceramide monolayers deposited at $30 \mathrm{mN} / \mathrm{m}$. Images $\mathrm{C}$ and $\mathrm{D}$ are obtained after smoothing of the $\mathrm{m} / \mathrm{z} 2$ and 281 images shown in $\mathrm{A}$ and $\mathrm{B}$, respectively. The pixel size for images $\mathrm{A}-\mathrm{D}$ is $404 \mathrm{~nm}$ and for images $\mathrm{E}$ and $\mathrm{F}$ is $175 \mathrm{~nm}$. The experimental resolution is estimated to be $\sim 300$ $\mathrm{nm}$.

is localized almost exclusively in the domains and is uniformly distributed throughout the domains. This is evident in both processed and unprocessed images (compare parts A and B of Figure 6 to parts $C$ and D of Figure 6 ) and is typical of a number of sample areas as shown by the smaller scale images in parts $\mathrm{E}$ and $\mathrm{F}$ of Figure 6. This is despite the observation that AFM images (data not shown) indicate that monolayers prepared with $\mathrm{C}_{16: 0^{-}} \mathrm{SM}-d_{31}$ have small raised areas within the large domains, similar to results for undeuterated SM. These results suggest that the domain heterogeneity is primarily due to variability in the distribution of ceramide, rather than SM. By contrast, monolayers containing $30 \% \mathrm{SM}-d_{31}$ at $10 \mathrm{mN} / \mathrm{m}$ showed both homogeneous and heterogeneous domains in ToF-SIMS images, depending on the sample area, and were not examined in detail.

The above results provide qualitative information on the distribution of the various lipids for ceramide-containing monolayers. However, it is not possible to use the relative intensities for individual ions as a direct measure of the lipid concentration because a number of studies have shown that ion yields are not proportional to concentrations due to matrix effects. For example, differences in ion yields for DPPC in the liquid-expanded and the liquid-condensed phases are due to variations in both lipid densities and ease of fragmentation in the two phases. ${ }^{58}$ Similarly, changes in the monolayer film density and substrate as well as addition of proton-donating molecules such as cholesterol have all been shown to affect the DPPC ion yield. In this study, the use of self-assembled monolayers on gold rather than silicon as a substrate and the addition of chol increased the ion yield. ${ }^{59}$ Freeze-dried POPC bilayers were shown to give 5-10 times enhancement of molecular ion yields compared to POPC monolayers or disordered films of POPC ${ }^{60}$ A recent publication from the Winograd group has taken a quantitative approach to evaluating matrix effects for ternary lipid mixtures containing $\mathrm{POPC} / \mathrm{SM} / \mathrm{chol}$ mixtures. ${ }^{43}$ Here, the signal from the underlying gold substrate was used as a matrix reference ion for the calculation of relative sensitivity factors (RSFs) for individual lipid monolayers and the various binary and ternary lipid mixtures. On the basis of these results, the relative ion yields are approximately two times higher for SM and POPC in mixed monolayers than for single component monolayers, whereas the yield for chol is lower by a factor of 2 . However, the RSF values for the various possible binary or ternary mixtures are less dependent on the lipid ratios (variations of $<30 \%$ ); for example the RSF for SM is only $11 \%$ higher in 2:3 POPC/SM monolayers than in 2:1 $\mathrm{SM} /$ chol monolayers.

On the basis of the above considerations, we have used the relative ratio for the $\mathrm{m} / \mathrm{z} 2$ deuterium signal from ceramide- $d_{31}$ to show that ceramide is concentrated in small raised islands within the ordered domains. Specifically, we estimate the ratio of ceramide in the small raised islands relative to the surrounding domains for the images shown in part B of Figure 4 and part B of 5. We obtain values of this ratio to be 2.6 and 4 for monolayers transferred at 10 and $30 \mathrm{mN} / \mathrm{m}$, respectively. Here, we have ignored any ion yield variation due to matrix effects because the relative sensitivity factor for SM does not vary much between $\mathrm{POPC} / \mathrm{SM}$ and SM/chol monolayers. ${ }^{43}$ We conclude that the ceramide concentration in the DOPC phase is negligible on the basis of our inability to detect a significant deuterium signal. Similarly, we estimate that the yield of SM in the DOPC phase is low. By contrast, DOPC is detected in both domains and fluid phase, although due to the relatively low intensity of the oleate signal and possible matrix effects we have not attempted to estimate the ratios in the two phases. On the basis of the literature observation that the yield of POPC is significantly higher in the ordered phase, ${ }^{43}$ the relative ion ratios for DOPC are likely to be an overestimate of the domain/fluid phase partitioning ratio.

\section{Discussion}

Replacement of SM by ceramide in ternary lipid mixtures of DOPC/ESM/cholesterol leads to significant changes in morphology of the original SM and cholesterol-rich ordered domains as visualized by AFM. The domains become heterogeneous with two distinct heights. The higher phase comprises a larger fraction of the domains at the higher ceramide concentration, suggesting that it is ceramide-rich, presumably a gel phase. These results agree with a number of studies that have indicated that ceramide promotes monolayer heterogeneity and phase separation via the formation of highly ordered gel phase domains, consistent with its relatively high melting temperature $\left(T_{\mathrm{M}}=90 \mathrm{C}\right) .{ }^{61}$ For example, phase separation to give ceramide and SM-enriched domains has been observed when ceramide is added to sphingomyelin monolayers, either by direct incorporation or enzymatic generation with sphingomyelinase. ${ }^{35,36}$ Similarly, ceramide-

(59) Sostarecz, A. G.; Cannon, D. M.; McQuaw, C. M.; Sun, S.; Ewing, A. G.; Winograd, N. Langmuir 2004, 20, 4926-4932.

(60) Prinz, C.; Hook, F.; Malm, J.; Sjovall, P. Langmuir 2007, 23, 8035-8041.

(61) Shah, J.; Atienza, J. M.; Duclos, R. I.; Rawlings, A. V.; Dong, Z.; Shipley, G. G. J. Lipid Res. 1995, 36, 1936. 
enriched domains have been observed in mixed monolayers with either cholesterol or phosphatidylcholine..$^{29-34}$ Although the earlier results make it clear that ceramide promotes phase separation in simple binary mixtures, the present study has visualized directly the effects of ceramide on domain heterogeneity in monolayers with coexisting liquid phases and has correlated monolayer morphology with lipid composition.

Ceramide-promoted phase separation has been observed in vesicles prepared from binary and ternary lipid mixtures. ${ }^{26,24,62}$ Recent studies of ceramide incorporation in supported lipid bilayers prepared from ternary lipid mixtures have used AFM and fluorescence to detect heterogeneous liquid-ordered domains that were assigned to ceramide enriched regions of the membrane. ${ }^{18,27,28,63}$ The ceramide-enriched domains were significantly thicker $(0.5-1.5 \mathrm{~nm})$ than the surrounding domain. These results were rationalized on the basis of the formation of a ceramide gel phase that is thicker and more rigid, both of which contribute to an apparent increase in height. The AFM data for the monolayers examined here indicates that there is a relatively small height difference between ceramide-rich and ceramidedepleted regions, consistent with the competing effects of a smaller lipid headgroup but a more tightly packed and thicker ceramiderich gel phase.

The ToF-SIMS imaging of monolayers for which a fraction of SM has been replaced by ceramide provides more information on the lipid distribution within domains and fluid phase than can be obtained from either AFM or fluorescence. The results demonstrate that ceramide and SM are detected almost exclusively in the domains, whereas DOPC is predominantly in the surrounding fluid phase as expected. Furthermore, ceramide is concentrated in small subdomains within the ordered domains for monolayers containing ceramide- $d_{31}$. The intensity of the ceramide-derived deuterium signal is much higher in the ceramide-enriched areas than in the remainder of the domains and no ceramide signal is detected in the surrounding DOPCrich fluid phase. It is important to note that the ceramide heterogeneity is clearly evident in unprocessed ToF-SIMS images, although the processed images are more informative for the DOPC oleate signal. Although matrix effects complicate the measurement of lipid ratios from ion intensities, we have estimated an enrichment of 2.6 and 4 fold for ceramide in the small islands compared to the remainder of the ordered domains at 10 and 30 $\mathrm{mN} / \mathrm{m}$, respectively. The larger enrichment of ceramide at high surface pressure is consistent with tightly packed ordered domains.

Qualitatively different results are obtained for samples containing deuterated SM. In this case, there is also a strong preference of SM for the ordered domains but it is uniformly distributed throughout the domain. Several recent studies have concluded that addition of ceramide to ordered SM and cholesterol-rich domains in bilayer membranes leads to expulsion

(62) Castro, B. M.; de Almeida, R. F. M.; Silva, L. C.; Federov, A.; Prieto, M. Biophys. J. 2007, 93, 1639-1650.

(63) Chiantia, S.; Kahya, N.; Schwille, P. Langmuir 2007, 23, 7659-7665. of cholesterol into the fluid phase. ${ }^{16,18,19}$ Although phase boundaries differ between monolayers and bilayers, ${ }^{50}$ we hypothesize that a similar loss of chol from the domains may lead to ceramide-SM enriched islands and SM-chol rich regions of the domain. This could explain the observation that SM is uniformly distributed throughout the domains, whereas ceramide is clustered in specific regions. In principle, ToF-SIMS mapping can also provide information on changes in chol distribution after ceramide addition. However, as noted in the Results section, we were unable to observe chol in the present studies. Previous work has shown that $30 \%$ chol- $d_{7}$ is sufficient to monitor chol in pulmonary surfactant monolayers, although the signals are relatively weak. ${ }^{42}$ A number of studies from the Winograd group have used ToF-SIMS to monitor chol distribution in lipid monolayers deposited on self-assembled monolayers on gold and in cell membranes. ${ }^{43-45,59,64,65}$ The higher sensitivity for cholesterol detection in their monolayer studies may reflect matrix effects due to either the different support used (self-assembled monolayers on gold) or to changes in ioniziation efficiency as a result of lipid composition. Because the lipid mixtures used in the present work and a published study of a similar ternary lipid mixture (SM/POPC/chol) ${ }^{43}$ differ only in the use of DOPC versus POPC and have similar amounts of chol (20 and 23\%), we hypothesize that the difference in support is more likely to be the source of our inability to detect chol.

In summary, the present study demonstrates that replacement of SM by ceramide in ternary lipid mixtures that are used as models for membrane rafts leads to increased domain order and heterogeneity. Incorporation of ceramide leads to irregularly shaped, heterogeneous domains with raised subdomains that are assigned to a ceramide-enriched gel phase, as confirmed by chemical mapping using ToF-SIMS. This work supports conclusions of several other recent studies demonstrating the utility of mass spectrometry imaging for identifying the chemical composition of domains in phase-separated supported lipid monolayers and bilayers. ${ }^{40,42-44}$ It also highlights the complementary information that can be obtained by correlating topographic imaging with mass spectrometry and fluorescence imaging. We believe that application of a similar multimodal imaging approach has potential for understanding the changes in lipid distribution that lead to membrane restructuring following enzymatic generation of ceramide in monolayers or supported lipid bilayers. $^{35,36}$

Supporting Information Available: Isotherms and mass spectra for monolayers. This material is available free of charge via the Internet at http://pubs.acs.org.

\section{LA8007552}

(64) McQuaw, C. M.; Sostarecz, A. G.; Zheng, L.; Ewing, A. G.; Winograd, N. Langmuir 2005, 21, 807-813.

(65) Ostrowski, S. G.; Kurczy, M. E.; Roddy, T. P.; Winograd, N.; Ewing, A. G. Anal. Chem. 2007, 79, 3554-3560. 